Volume 133, Number 10, Pages 3103-3108

S 0002-9939(05)07840-8

Article electronically published on May 4, 2005

\title{
CAN A LARGE CARDINAL BE FORCED FROM A CONDITION IMPLYING ITS NEGATION?
}

\author{
ARTHUR W. APTER AND GRIGOR SARGSYAN \\ (Communicated by Carl G. Jockusch, Jr.)
}

\begin{abstract}
In this note, we provide an affirmative answer to the title question by giving two examples of cardinals satisfying conditions implying they are non-Rowbottom which can be turned into Rowbottom cardinals via forcing. In our second example, our cardinal is also non-Jonsson.
\end{abstract}

A well-known phenomenon is that under certain circumstances, it is possible to force over a given model $V$ of ZFC containing a cardinal $\kappa$ satisfying a large cardinal property $\varphi(\kappa)$ to create a universe $\bar{V}$ in which $\varphi(\kappa)$ no longer holds, yet over which $\varphi(\kappa)$ can be resurrected via forcing. A folklore example of this is provided by supposing that $V \vDash$ "ZFC $+\mathrm{GCH}+\kappa$ is measurable". If $\mathbb{P}$ is defined as the reverse Easton iteration of length $\kappa$ which adds a Cohen subset to each inaccessible cardinal below $\kappa$, then in $V^{\mathbb{P}}, \kappa$ is no longer measurable, by, e.g., a simpler version of the argument given in Lemma 2.4 of [1]. However, if one forces over $V^{\mathbb{P}}$ by adding a Cohen subset to $\kappa$, then the standard reverse Easton arguments show that $\kappa$ 's measurability has been resurrected, since the entire forcing can now be viewed as the length $\kappa+1$ reverse Easton iteration over $V$ that adds a Cohen subset to each inaccessible cardinal less than or equal to $\kappa$.

In this note, we consider a different, stronger phenomenon. Specifically, we examine the following.

Question. Suppose $\varphi$ is a given large cardinal property. Is it possible to find a formula $\psi$ in one free variable in the language of set theory and a cardinal $\kappa$ such that $\psi(\kappa)$ holds, ZFC $\vdash " \forall \lambda[\psi(\lambda) \Longrightarrow \neg \varphi(\lambda)] "$, yet there is a partial ordering $\mathbb{P}$ such that $\vdash_{\mathbb{P}} \varphi(\kappa)$ ?

We show that the answer to our Question is yes for $\varphi$ the large cardinal property of Rowbottomness by proving the following theorem.

Received by the editors August 30, 2003 and, in revised form, February 14, 2004 and June 15, 2004

2000 Mathematics Subject Classification. Primary 03E02, 03E35, 03E55.

Key words and phrases. Jonsson cardinal, Rowbottom cardinal, strongly compact cardinal.

Both authors wish to thank the CUNY Research Foundation for having provided partial support for this research via the first author's PSC-CUNY Grant 64455-00-33, under which the second author was a research assistant. In addition, we wish to thank the referee and Carl Jockusch, the communicating editor, for helpful comments, corrections, and suggestions which have been incorporated into this version of the paper and which have considerably improved the presentation of the material contained herein.

(C)2005 American Mathematical Society Reverts to public domain 28 years from publication 
Theorem. (1) There is a formula $\psi_{1}(x)$ in one free variable such that ZFC $\vdash$ " $\psi_{1}(\kappa) \Longrightarrow\left[\kappa\right.$ is not Rowbottom, yet for some partial ordering $\mathbb{P}, \mathbb{F}_{\mathbb{P}}$ " $\kappa$ is a Rowbottom cardinal carrying a Rowbottom filter"] for all cardinals $\kappa$ ".

(2) There is a formula $\psi_{2}(x)$ in one free variable such that $\mathrm{ZFC} \vdash$ " $\psi_{2}(\kappa) \Longrightarrow[\kappa$ is not Jonsson, yet for some partial ordering $\mathbb{P}, \Vdash_{\mathbb{P}}$ " $\kappa$ is a Rowbottom cardinal carrying a Rowbottom filter"] for all cardinals $\kappa "$.

Note that $\mathcal{F}$ is a Rowbottom filter for $\kappa$ if for any $\lambda<\kappa$ and $f:[\kappa]^{<\omega} \rightarrow \lambda$, there is some $A \in \mathcal{F}$ such that $\left|f^{\prime \prime}[A]^{<\omega}\right| \leq \omega$ (where as usual, $[\kappa]^{<\omega}$ is the set of both empty and non-empty finite sequences of elements of $\kappa$, where each non-empty finite sequence is written in increasing order).

In other words, there is a cardinal $\kappa_{1}$ which satisfies a condition making it automatically non-Rowbottom, yet which can be turned into a Rowbottom cardinal carrying a Rowbottom filter via forcing. Further, there is another cardinal $\kappa_{2}$ which satisfies a condition making it automatically non-Jonsson, yet which can be turned into a Rowbottom cardinal carrying a Rowbottom filter via forcing. This is in sharp contrast to the example given in the first paragraph of this note and the examples to be given in the next to last paragraph of this note. There, one simply has a model $V$, a large cardinal property $\varphi$, a cardinal $\kappa$, and a partial ordering $\mathbb{P}$ such that $V \vDash \neg \varphi(\kappa)$ and $V^{\mathbb{P}} \vDash \varphi(\kappa)$. In the Theorem, however, one has that for every model $V$, for the appropriate $\varphi$ and $\psi$, if $V \vDash \psi(\kappa)$, then both $V \vDash \neg \varphi(\kappa)$ and there is a partial ordering $\mathbb{P} \in V$ with $V^{\mathbb{P}} \vDash \varphi(\kappa)$. This provides a strong positive answer to our Question.

Before beginning the proof of the Theorem, we briefly mention that we assume throughout a basic understanding of large cardinals and forcing. We refer readers to [5] for anything left unexplained.

We turn now to the proof of clause (1) of the Theorem.

Proof. To prove clause (1) of the Theorem, let $\kappa_{1}$ be a singular limit of $\delta$ measurable cardinals, where $\delta<\kappa_{1}$ is a regular uncountable cardinal. Take $\psi_{1}(x)$ as the formula in one free variable asserting this fact. Let $\mathbb{P}$ be the partial ordering which collapses $\delta$ to $\omega$. After forcing with $\mathbb{P}$, by the Lévy-Solovay results [9], a final segment of measurable cardinals below $\kappa_{1}$ remains measurable. Further, $\kappa_{1}$ can now be written as a limit of $\omega$ measurable cardinals. Therefore, by a theorem of Prikry (see [5], Theorem 8.7, page 90), $\kappa_{1}$ has become a Rowbottom cardinal carrying a Rowbottom filter 1 However, it is a theorem of ZFC (see [5], Exercise 8.6, page 90) that originally, since $\kappa_{1}$ had uncountable cofinality, $\kappa_{1}$ was not a Rowbottom cardinal. Thus, we have turned a cardinal satisfying a condition making it nonRowbottom via forcing into a Rowbottom cardinal, thereby proving clause (1) of the Theorem.

We note that since the partial ordering $\mathbb{P}$ defined above has cardinality less than $\kappa_{1}$, our proof of clause (1) of the Theorem illustrates the interesting occurrence that it is possible for small forcing to turn a provably non-Rowbottom cardinal into a Rowbottom cardinal.

We turn now to the proof of clause (2) of the Theorem.

\footnotetext{
${ }^{1}$ Although Theorem 8.7 of [5] does not explicitly state that a limit of $\omega$ measurable cardinals carries a Rowbottom filter, this is clear from the proof presented in [5].
} 
Proof. To prove clause (2) of the Theorem, let $\kappa_{2}$ be the least cardinal which is both regular and a limit of strongly compact cardinals. Take $\psi_{2}(x)$ as the formula in one free variable asserting this fact. We immediately have the following lemma.

Lemma 1. $\kappa_{2}$ is not a Jonsson cardinal.

Proof. If $\kappa_{2}$ were a Jonsson cardinal, then since $\kappa_{2}$ is strongly inaccessible, it is a theorem of Shelah (see [10], Chapters 3 and 4) that $\kappa_{2}$ must also be a Mahlo cardinal. Since the set $C$ of strongly compact cardinals below $\kappa_{2}$ is unbounded in $\kappa_{2}$, by the Mahloness of $\kappa_{2}$, the collection $C^{\prime}$ of limit points of $C$ must contain a strongly inaccessible cardinal. This means that $\kappa_{2}$ is not the least cardinal which is both regular and a limit of strongly compact cardinals, a contradiction which completes the proof of Lemma 1

Now that we know that $\kappa_{2}$ is not a Jonsson cardinal, we define the partial ordering $\mathbb{P}$ which turns $\kappa_{2}$ into a Rowbottom cardinal carrying a Rowbottom filter. $\mathbb{P}$ is a version of the "modified Prikry forcing" given in [4] and [2] but defined using more than one ultrafilter. More specifically, since $\kappa_{2}$ is both regular and a limit of strongly compact cardinals, let $\left\langle\kappa_{\alpha}^{*}: \alpha<\kappa_{2}\right\rangle$ be a sequence of strongly compact cardinals whose limit is $\kappa_{2}$, and let $\left\langle\mathcal{U}_{\alpha}: \alpha<\kappa_{2}\right\rangle$ be a sequence of ultrafilters such that each $\mathcal{U}_{\alpha}$ is a $\kappa_{\alpha}^{*}$-additive uniform ultrafilter over $\kappa_{2} . \mathbb{P}$ may now be defined as the set of all finite sequences of the form $\left\langle\alpha_{1}, \ldots, \alpha_{n}, f\right\rangle$ satisfying the following properties.

(1) $\left\langle\alpha_{1}, \ldots, \alpha_{n}\right\rangle \in\left[\kappa_{2}\right]^{<\omega}$.

(2) $f$ is a function having domain

$$
\begin{aligned}
T_{\alpha_{1}, \ldots, \alpha_{n}}=\left\{\left\langle\beta_{1}, \ldots, \beta_{m}\right\rangle \in\right. & {\left[\kappa_{2}\right]^{<\omega}: } \\
& \left.\left\langle\alpha_{1}, \ldots, \alpha_{n}\right\rangle \text { is an initial segment of }\left\langle\beta_{1}, \ldots, \beta_{m}\right\rangle\right\}
\end{aligned}
$$

such that $f\left(\left\langle\beta_{1}, \ldots, \beta_{m}\right\rangle\right) \in \mathcal{U}_{\beta_{m}}$.

The ordering on $\mathbb{P}$ is given by

$$
\left\langle\beta_{1}, \ldots, \beta_{m}, g\right\rangle \geq\left\langle\alpha_{1}, \ldots, \alpha_{n}, f\right\rangle
$$

$\left(\left\langle\beta_{1}, \ldots, \beta_{m}, g\right\rangle\right.$ is stronger than $\left.\left\langle\alpha_{1}, \ldots, \alpha_{n}, f\right\rangle\right)$ iff the following criteria are met.

(1) $\left\langle\alpha_{1}, \ldots, \alpha_{n}\right\rangle$ is an initial segment of $\left\langle\beta_{1}, \ldots, \beta_{m}\right\rangle$.

(2) For $i=n+1, \ldots, m, \beta_{i} \in f\left(\left\langle\alpha_{1}, \ldots, \alpha_{n}, \ldots, \beta_{i-1}\right\rangle\right)$.

(3) For every $s \in \operatorname{dom}(g)$ (which must be a subset of $\operatorname{dom}(f)), g(s) \subseteq f(s)$.

Lemma 2. Given any formula $\varphi$ in the forcing language with respect to $\mathbb{P}$ and any condition $\left\langle\alpha_{1}, \ldots, \alpha_{n}, f\right\rangle \in \mathbb{P}$, there is a condition

$$
\left\langle\alpha_{1}, \ldots, \alpha_{n}, f^{\prime}\right\rangle \geq\left\langle\alpha_{1}, \ldots, \alpha_{n}, f\right\rangle
$$

deciding $\varphi$.

Proof. The proof of Lemma 2 is essentially the same as the proof of Lemma 4.1 of 4] or Lemma 1.1 of [2], taking into account that different ultrafilters are used in the definition of $\mathbb{P}$. We follow the proofs of these lemmas almost verbatim, making the necessary minor changes where warranted. Specifically, let $s=\left\langle\alpha_{1}, \ldots, \alpha_{n}\right\rangle$. For any $t \in T_{s}$, call $t$ sufficient if, for some $g,\langle t, g\rangle \| \varphi$. For $t$ sufficient, let $g_{t}$ be a witness, with $g_{t}(r)=\kappa_{2}$ for all $r \in \operatorname{dom}\left(g_{t}\right)$ if $t$ is not sufficient. If $s$ is sufficient, 
then we are done. If not, then for any $t \in T_{s}$, sufficient or otherwise, one of the sets

$$
\begin{gathered}
X_{t}=\left\{\alpha<\kappa_{2}: \exists g\left[\left\langle t^{\frown} \alpha, g\right\rangle \Vdash \varphi\right]\right\}, \\
Y_{t}=\left\{\alpha<\kappa_{2}: \exists g\left[\left\langle t^{\frown} \alpha, g\right\rangle \Vdash \neg \varphi\right]\right\}, \text { or } \\
Z_{t}=\left\{\alpha<\kappa_{2}: \forall g\left[\left\langle t^{\frown} \alpha, g\right\rangle \text { does not decide } \varphi\right]\right\}
\end{gathered}
$$

is an element of $\mathcal{U}_{\max (t)}$. Let $A_{t}$ be that set, and for $i \leq \operatorname{length}(t)$, let $t\lceil i$ be the first $i$ members of $t$. For $t \in T_{s}$, define $f^{\prime}$ by

$$
f^{\prime}(t)=f(t) \cap \bigcap_{n \leq i \leq \operatorname{length}(t)} g_{t \uparrow i}(t) \cap A_{t} .
$$

Note that by the definition of $\mathbb{P}, f^{\prime}(t) \in \mathcal{U}_{\max (t)}$, which means that $\left\langle s, f^{\prime}\right\rangle$ is a well-defined member of $\mathbb{P}$ extending $\langle s, f\rangle$.

Now, let $t$ be sufficient and of minimal length $m+1>n$, with $\left\langle t, f^{\prime \prime}\right\rangle \geq\left\langle s, f^{\prime}\right\rangle$ and $f^{\prime \prime}=f^{\prime} \uparrow T_{t}$. Let $t^{\prime}$ be the sequence $t$ without its last element. It then follows that $A_{t^{\prime}}$ must be either $X_{t^{\prime}}$ or $Y_{t^{\prime}}$, so we suppose without loss of generality that $A_{t^{\prime}}=X_{t^{\prime}}$. It must be the case that $\left\langle t^{\prime}, f^{\prime}\left\lceil T_{t^{\prime}}\right\rangle \Vdash \varphi\right.$, since if some extension $\left\langle t^{\prime \prime}, g^{\prime}\right\rangle \Vdash \neg \varphi$, such a condition must add elements to $t^{\prime}$, since $t^{\prime}$ isn't sufficient. The first element added to $t^{\prime}, \alpha$, must come from $X_{t^{\prime}}$, yielding a condition $\left\langle t^{\prime} \neg\{\alpha\} \frown u, g^{\prime}\right\rangle \Vdash \neg \varphi$. However, by construction,

$$
\left\langle t^{\prime}\left\{\{\alpha\} \frown u, g^{\prime}\right\rangle \geq\left\langlet ^ { \prime } \left\{\{\alpha\}, f^{\prime}\left|T_{t^{\prime}}-\{\alpha\}\right\rangle \geq\left\langle t^{\prime} \frown\{\alpha\}, g_{t^{\prime}}-\{\alpha\}\right\rangle \Vdash \varphi,\right.\right.\right.
$$

which is a contradiction. Thus, $\left\langle t^{\prime}, f^{\prime}\left\lceil T_{t^{\prime}}\right\rangle \| \varphi\right.$, which contradicts the minimality of the length of $t$ for sufficiency. This completes the proof of Lemma 2 ,

Lemma 3. Forcing with $\mathbb{P}$ adds no new subsets to any $\delta<\kappa_{2}$.

Proof. Given $\delta<\kappa_{2}$, suppose that $p=\left\langle\alpha_{1}, \ldots, \alpha_{n}, f\right\rangle \Vdash$ " $\tau \subseteq \delta$ ". Without loss of generality, by extending $p$ if necessary, we also assume that $\kappa_{\alpha_{n}}^{*}>\delta$. Further, by Lemma 2 for each $\beta<\tau$, we let $\left\langle\alpha_{1}, \ldots, \alpha_{n}, f_{\beta}\right\rangle$ be such that $\left\langle\alpha_{1}, \ldots, \alpha_{n}, f_{\beta}\right\rangle \|$ " $\beta \in \tau "$.

Note that the domains of all of the $f_{\beta}$ 's for $\beta<\delta$ and $f$ are the same, namely $T_{\alpha_{1}, \ldots, \alpha_{n}}$. Therefore, by the choice of $p$ and the definition of $\mathbb{P}$, for each $s \in T_{\alpha_{1}, \ldots, \alpha_{n}}$, $f_{\beta}(s)$ and $f(s)$ lie in an ultrafilter $\mathcal{U}_{\max (s)}$ that is $\kappa_{\alpha_{n}}^{*}$-additive. This means that $g(s)=\bigcap_{\beta<\delta} f_{\beta}(s) \cap f(s)$ is such that $g(s) \in \mathcal{U}_{\max (s)}$, and $q=\left\langle\alpha_{1}, \ldots, \alpha_{n}, g\right\rangle$ is a well-defined element of $\mathbb{P}$ such that $q \geq p$ and $q$ decides the statement " $\beta \in \tau$ " for every $\beta<\delta$. Hence, forcing with $\mathbb{P}$ adds no new subsets to $\delta$. This completes the proof of Lemma 3

The proof of clause (2) of the Theorem now easily follows. The usual density arguments show that after forcing with $\mathbb{P}, \operatorname{cof}\left(\kappa_{2}\right)=\omega$. Further, by Lemma 3 since forcing with $\mathbb{P}$ adds no new bounded subsets to $\kappa_{2}$, after forcing with $\mathbb{P}, \kappa_{2}$ remains a limit of measurable cardinals. Therefore, once again by Prikry's theorem used in the proof of clause (1) of the Theorem, $\kappa_{2}$ is after forcing with $\mathbb{P}$ a Rowbottom cardinal carrying a Rowbottom filter. This completes the proof of clause (2) of the Theorem, and hence also completes the proof of the Theorem.

It is a theorem of ZFC (see [5], Theorem 8.7, page 90) that a $\kappa_{1}$ as in clause (1) of the Theorem is Jonsson (or more specifically, is $\delta^{+}$Rowbottom). Thus, in clause (1) of the Theorem, we actually have that a cardinal which is both Jonsson and partially Rowbottom is turned into a fully Rowbottom cardinal carrying a 
Rowbottom filter via small forcing. This contrasts with what occurs in clause (2) of the Theorem, where a cardinal satisfying a condition making it automatically non-Jonsson is transformed into a Rowbottom cardinal carrying a Rowbottom filter. Further, as readers may verify for themselves, our methods of proof yield that the least cardinal $\lambda$ which is both regular and a limit of cardinals which are $\lambda$ strongly compact suffices as the $\kappa_{2}$ of clause (2) of the Theorem.

We take this opportunity to make some remarks concerning relative consistency results related to the Theorem. First, as Devlin has shown in [3], it is consistent, relative to the existence of a Ramsey cardinal, for the least Jonsson cardinal $\kappa$ not to be Rowbottom. Since Kleinberg has shown (see [6], 7], or [5], Propositions 8.15(c) and 10.18, pages 95 - 96 and 128 - 129 respectively) that under these circumstances, $\kappa$ is $\delta$ Rowbottom for some $\delta<\kappa$ and can be transformed into a Rowbottom cardinal via a forcing having size less than $\kappa$, we have that the existence of a nonRowbottom Jonsson cardinal which can be changed into a Rowbottom cardinal via small forcing is consistent relative to a Ramsey cardinal. Further, Woodin has shown (see page 60 of [8]) that the stationary tower forcing can be used, assuming either a proper class of measurable cardinals (for class forcing), or an inaccessible limit of measurable cardinals with a Woodin cardinal above it (for set forcing), to change the cofinality of the least inaccessible limit $\kappa$ of measurable cardinals to $\omega$ (or indeed, to any regular cardinal $\delta<\kappa$ ) without adding bounded subsets to $\kappa$. This thereby gives that the existence of a non-Jonsson cardinal which can be turned into a Rowbottom cardinal carrying a Rowbottom filter via forcing is consistent relative to assumptions much weaker than even the existence of a cardinal $\lambda$ which is $\lambda^{+}$ strongly compact. However, we emphasize again that these results, like the one given in the first paragraph of this note, are only relative consistency results. This is in sharp contrast to the Theorem, which is an outright ZFC theorem, and thus provides a much stronger phenomenon.

In conclusion, we ask if there are other large cardinal or combinatorial properties that provide answers to our main Question for a particular cardinal $\kappa$. If so, can these properties be added by a forcing which doesn't add bounded subsets to $\kappa$ and doesn't collapse cardinals, or by a forcing which is small relative to $\kappa$ ? Is there such a property implying that $\kappa$ is a regular cardinal?

\section{REFERENCES}

[1] A. Apter, J. D. Hamkins, "Exactly Controlling the Non-Supercompact Strongly Compact Cardinals", Journal of Symbolic Logic 68, 2003, 669-688. MR1976597 (2004b:03075)

[2] A. Apter, J. Henle, "Relative Consistency Results via Strong Compactness", Fundamenta Mathematicae 139, 1991, 133-149. MR1150596 (93h:03071)

[3] K. Devlin, "Some Weak Versions of Large Cardinal Axioms", Annals of Mathematical Logic 5, 1973, 291-325. MR0363906|(51:161)

[4] J. Henle, "Partition Properties and Prikry Forcing", Journal of Symbolic Logic 55, 1990, 938-947. MR 1071307 (91k:03132)

[5] A. Kanamori, The Higher Infinite, Springer-Verlag Publishing Company, Berlin and New York, 1994. MR 1321144 (96k:03125)

[6] E. Kleinberg, "Rowbottom Cardinals and Jonsson Cardinals are Almost the Same", Journal of Symbolic Logic 38, 1973, 423-427. MF0337616 (49:2385)

[7] E. Kleinberg, "The Equiconsistency of Two Large Cardinal Axioms", Fundamenta Mathematicae 102, 1979, 81-85. MR0525930|(80d:03056)

[8] P. Larson, The Stationary Tower, University Lecture Series, vol. 32, American Mathematical Society, Providence, RI, 2004. MR2069032 
[9] A. Lévy, R. Solovay, "Measurable Cardinals and the Continuum Hypothesis", Israel Journal of Mathematics 5, 1967, 234-248. MR0224458 (37:57)

[10] S. Shelah, Cardinal Arithmetic, Oxford Logic Guides 29, Clarendon Press, Oxford, 1994. MR1318912 (96e:03001)

Department of Mathematics, Baruch College of CUNy, New York, New York 10010

E-mail address: awabb@cunyvm.cuny.edu

$U R L:$ http://faculty. baruch. cuny.edu/apter

Group in Logic and the Methodology of Science, University of California, Berkeley, CALIFORNIA 94720

E-mail address: grigor@math.berkeley.edu

$U R L:$ http://math.berkeley.edu/ ${ }^{\sim}$ grigor 